Original Article

\title{
Accuracy of ultrasound diagnosis of nonalcoholic fatty liver disease in patients with classes II and III obesity: A pathological image study
}

\author{
Gabriel Leivas $^{\mathrm{a}}$, Clara K. Maraschin ${ }^{\mathrm{a}}$, Carina A. Blume ${ }^{\mathrm{b}}$, Gabriela H. Telo ${ }^{\mathrm{c}, \mathrm{d}}$, Manoel R. \\ M. Trindade ${ }^{\mathrm{e}}$, Eduardo N. Trindade ${ }^{\mathrm{e}}$, Vinicius V. Diemen ${ }^{\mathrm{e}}$, Carlos Thadeu S. Cerski ${ }^{\mathrm{f}}$, Beatriz \\ D. Schaan ${ }^{\mathrm{b}, \mathrm{d}, \mathrm{g}, *}$
}

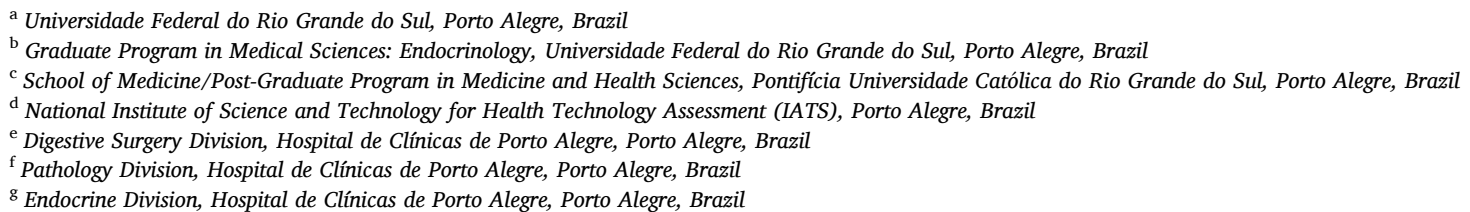

\section{A R T I C L E I N F O}

\section{Keywords:}

Bariatric surgery

Nonalcoholic fatty liver disease

Ultrasound

Liver biopsy

\begin{abstract}
A B S T R A C T
Liver biopsy is the gold standard method to diagnose nonalcoholic fatty liver disease (NAFLD). However, ultrasound is widely recommended as the first-line imaging test for individuals with suspected NAFLD. This study aimed to estimate the accuracy of ultrasound as a screening test for NAFLD compared to liver biopsy in a cohort of patients with class II and III obesity undergoing bariatric surgery. This retrospective study included patients undergoing Roux-en-Y gastric bypass in southern Brazil between 2010 and 2019 who were screened for NAFLD with both ultrasound and liver biopsy. All samples were collected by a core biopsy needle and were analyzed by the same pathologist. Sensitivity, specificity, and positive and negative predictive values of ultrasound were estimated. The final database included 227 patients, mostly female (84\%) and white (83.6\%), with a mean age of $42.5 \pm 10.2$ years and a mean preoperative body mass index of $49.5 \pm 8.4 \mathrm{~kg} / \mathrm{m}^{2}$. A total of 153 subjects $(67.4 \%)$ were diagnosed with NAFLD through liver biopsies: 41 (18\%) had fatty liver and 112 (49.3\%) had nonalcoholic steatohepatitis. Ultrasound sensitivity was $88.9 \%$ and specificity was $44.6 \%$. Positive and negative predictive values were $76.8 \%$ and $66.0 \%$, respectively. Positive likelihood ratio was 1.6 (95\% CI 1.30-1.98), and negative likelihood ratio was 0.25 (95\% CI 0.15-0.42). Therefore, approximately three every four subjects with an ultrasound suggesting NAFLD were true positives. Ultrasound showed a good sensitivity in detecting NAFLD in patients with class II and III obesity.
\end{abstract}

\section{Introduction}

Nonalcoholic fatty liver disease (NAFLD) is an increasingly common cause of chronic liver disease [1]. The current prevalence of NAFLD is estimated to be about one-quarter in adults worldwide, with geographic variations that indicate highest prevalences in the Middle East and South America [2]. The prevalence is estimated to be substantially higher among individuals with type 2 diabetes mellitus and obesity $-60 \%$ and
$90 \%$, respectively $[3,4]$. NAFLD has a broad spectrum of severity, ranging from nonalcoholic fatty liver (NAFL) to nonalcoholic steatohepatitis (NASH). If not effectively treated, NASH can lead to advanced cirrhosis and hepatocellular carcinoma [5]. The complications of NASH are expected to increase with the growing epidemic of type 2 diabetes mellitus and obesity worldwide [6].

Liver biopsy is recognized as the gold standard method to diagnose NAFLD. However, the risks of performing such an invasive procedure

\footnotetext{
* Corresponding author at: Serviço de Endocrinologia do Hospital de Clínicas de Porto Alegre, Rua Ramiro Barcelos, 2350 , Prédio 12 - $4^{\circ}$ andar, Porto Alegre, RS 90035-003, Brazil.

E-mail addresses: gleivas@hcpa.edu.br (G. Leivas), claramaraschin@gmail.com (C.K. Maraschin), carinablume@hotmail.com (C.A. Blume), gtelo.

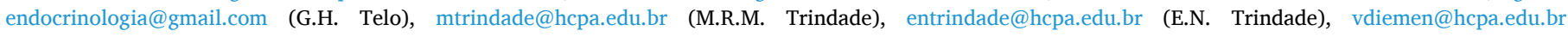
(V.V. Diemen), ccerski@hcpa.edu.br (C.T.S. Cerski), bschaan@hcpa.edu.br (B.D. Schaan).
} 
overcome the benefits in most cases [7]. Therefore, a more affordable and safer method for diagnosing NAFLD is needed [8]. Ultrasound is widely recommended as the first-line imaging test for individuals with suspected NAFLD [9]. Although a meta-analysis demonstrated that ultrasound has a pooled sensitivity of $84.8 \%$ and a specificity of $93.6 \%$ for moderate-to-severe steatosis compared with liver histology [10], ultrasound accuracy to diagnose NAFLD in patients with class II and III obesity is poorly described.

Although severe obesity is a well-known limitation to ultrasonographic assessment of the abdominal cavity, obese individuals are at major risk of developing NAFLD and its complications, which warrants some type of diagnostic test for NAFLD to be done [4]. Hence, intraoperative liver biopsies are commonly performed in patients undergoing bariatric surgery [11].

This study aimed to assess the accuracy of ultrasound as a screening test for NAFLD compared with liver biopsy in a cohort of patients with class II or III obesity undergoing bariatric surgery.

\section{Subjects}

All patients who underwent Roux-en-Y gastric bypass (RYGB) between January 2010 and December 2019 in a public, tertiary care, teaching hospital in Porto Alegre, southern Brazil, were assessed. The final sample included only those who underwent both ultrasound in the preoperative evaluation and liver biopsy during the bariatric surgery. Fig. 1 presents the flowchart of participants included in the final sample.

\section{Materials and methods}

\section{Study design}

This single-center retrospective cohort study was conducted to assess the accuracy of ultrasound in detecting NAFLD compared with liver biopsy in patients with class II or III obesity (body mass index [BMI] of $35.0-39.9 \mathrm{~kg} / \mathrm{m}^{2}$ and $\geq 40 \mathrm{~kg} / \mathrm{m}^{2}$, respectively) who underwent RYGB at Hospital de Clínicas de Porto Alegre (HCPA) between 2010 and 2019 [12]. Patients underwent ultrasound during the preoperative evaluation, and all biopsy samples were collected during the surgical procedure. This study was approved by the local Ethics Committee (2018-0088), and informed consent does not apply to this secondary analysis. This study is reported according to the STARD guidelines [13].

\section{Test methods}

Ultrasound was performed by different radiologists working at the same radiology service. The most common ultrasonographic criterion suggesting NAFLD was liver hyperechogenicity compared with the right kidney parenchyma [14], and ultrasound result was dichotomized in the analysis. Intraoperative liver biopsy samples were collected through a core biopsy needle (UNIGUN 18-gauge, $160 \mathrm{~mm}$; Medax Medical Devices). The Tru-Cut needle biopsy of the liver was the chosen method, and it was always performed on the right lobe. The specimens were fixed in formalin and embedded in paraffin for subsequent staining with hematoxylin/eosin and picrosirius. All samples were analyzed by the same pathologist. The radiologists and the pathologist were not blinded and had access to the patients' medical records.

Normal liver fat deposition was defined as the involvement of $5 \%$ or less of the parenchyma in liver histology. Steatosis was classified according to the percentage of fat infiltration of liver parenchyma as mild (6\%33\%), moderate (34\%-66\%), and severe (greater than 66\%) without evidence of hepatocellular injury in the form of ballooning degeneration of hepatocytes or evidence of fibrosis $[15,16]$. NASH was diagnosed in the presence of hepatic steatosis, ballooning degeneration, and inflammation [16].

Fasting plasma glucose $\geq 100 \mathrm{mg} / \mathrm{dL}$ was considered elevated. Total cholesterol levels were high when $\geq 200 \mathrm{mg} / \mathrm{dL}$, whereas high-density lipoprotein (HDL)-cholesterol was reduced when $<40 \mathrm{mg} / \mathrm{dL}$ in men and $<50 \mathrm{mg} / \mathrm{dL}$ in women. Triglyceride levels were elevated when $\geq 150$ $\mathrm{mg} / \mathrm{dL}$. Calculated low-density lipoprotein (LDL)-cholesterol was increased when $\geq 100 \mathrm{mg} / \mathrm{dL}$. Alanine transaminase (ALT) and aspartate transaminase (AST) were elevated when $>40 \mathrm{mg} / \mathrm{dL}$ and $>41 \mathrm{mg} / \mathrm{dL}$ in men and when $>32 \mathrm{mg} / \mathrm{dL}$ and $>33 \mathrm{mg} / \mathrm{dL}$ in women, respectively.

\section{Statistical analysis}

Quantitative data are shown as mean and standard deviation (SD) or median and interquartile range according to variable distribution. The Kolmogorov-Smirnov test was used to determine normality of study variables. Positive likelihood ratio, negative likelihood ratio, and accuracy are presented as mean and $95 \%$ confidence interval (CI). Categorical data are presented as frequencies.

Two-by-two tables were used to calculate sensitivity, specificity, positive and negative predictive values, and likelihood ratios (LRs). Ultrasound was considered the index test and liver histology was considered the reference standard. All statistical analyses were performed in IBM SPSS, version 22.0.

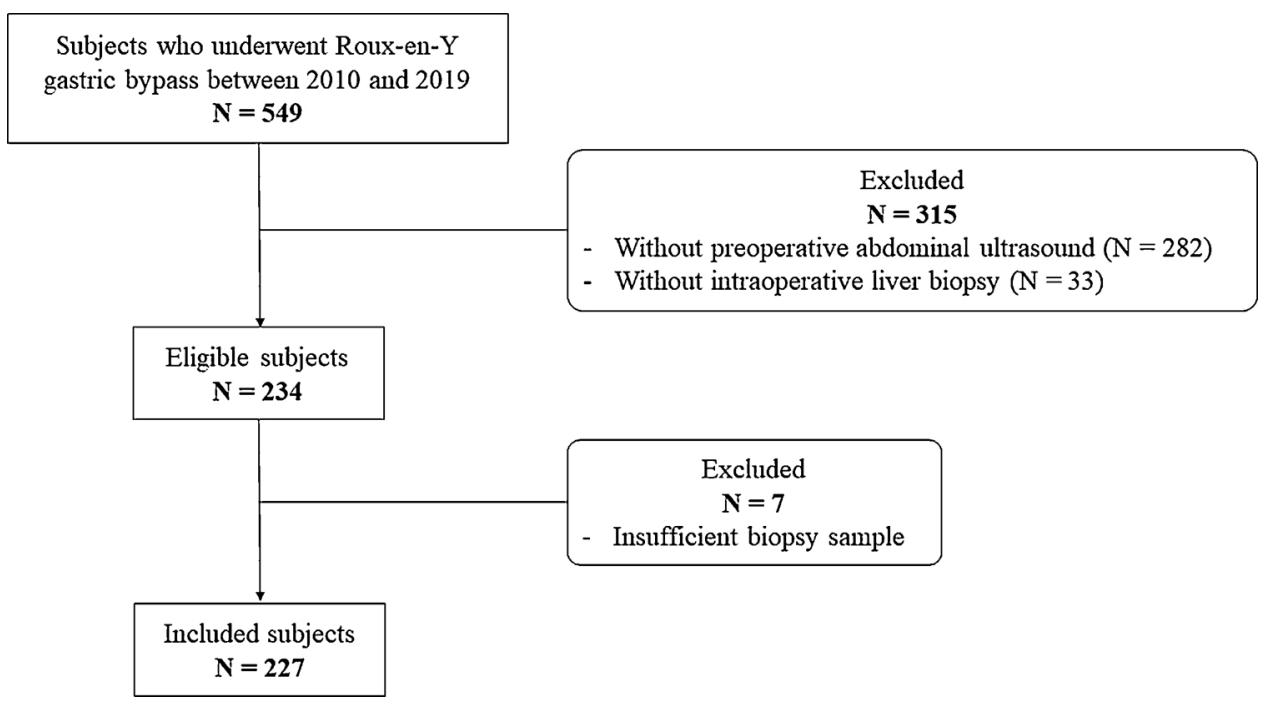

Fig. 1. Flowchart showing identification and selection of the study population $(\mathrm{N}=227)$. 


\section{Results}

The final database included 227 subjects (Fig. 1), mostly female $(83.7 \%)$ and white $(83.5 \%)$, with a mean age of $42.5 \pm 10.2$ years. Most patients were single $(53.2 \%)$ and had middle school or lower as level of education (51.6\%). Median waiting time for surgery was 38 (27-49) months. Self-reported average duration of obesity (defined as BMI $\geq 30$ $\mathrm{kg} / \mathrm{m}^{2}$ ) was $19.7 \pm 9.0$ years.

Table 1 presents baseline sociodemographic and clinical characteristics. The most prevalent obesity-related conditions were hypertension and dyslipidemia. Elevated fasting plasma glucose was found in $52.1 \%$ of the sample, whereas $34.5 \%$ had high total cholesterol levels. Reduced HDL-cholesterol was detected in $67 \%$, and $43.5 \%$ showed increased triglyceride levels. Calculated LDL-cholesterol was elevated in $60.8 \%$. Elevated AST and ALT were detected in $13.9 \%$ and $17.5 \%$, respectively.

Median interval between ultrasound and intraoperative biopsy was 9 (4-17) months. Ultrasound suggesting NAFLD was found in 177 subjects (78\%). Once 136 of those were true positives, the calculated positive predictive value was $76.8 \%$. Yet, 33 of the 50 patients with normal ultrasound were true negatives, which corresponds to a $66 \%$ negative predictive value.

A total of 153 subjects (67.4\%) were diagnosed with NAFLD through liver biopsies: 41 (18\%) had NAFL and $112(49.3 \%)$ had NASH. In both groups, mild fat deposition was the most common presentation (132 patients in total). Table 2 presents liver biopsy results stratified by the proportion of affected liver parenchyma.

Table 3 summarizes diagnostic accuracy for ultrasound. Estimated sensitivity and specificity were, respectively, $88.9 \%$ and $44.6 \%$. NAFLD prevalence detected through liver biopsy (67.4\%) corresponds to the pretest probability, as seen in Fig. 2. With a positive LR of 1.6 (95\% CI 1.30-1.98), approximately three every four subjects with an ultrasound suggesting NAFLD were true positives. Finally, with a negative LR of 0.25 (95\% CI 0.15-0.42), approximately two every three subjects with an ultrasound not suggesting NAFLD were true negatives.

\section{Discussion}

Current medical literature has significant gaps in the knowledge

Table 1

Participant baseline sociodemographic and clinical characteristics.

\begin{tabular}{ll}
\hline Characteristic & \\
\hline BMI $\left(\mathrm{kg} / \mathrm{m}^{2}\right)$ & $49.4 \pm 8.5$ \\
Excess weight (kg) & $64.9 \pm 24.2$ \\
Abdominal circumference (cm) & $134.5 \pm 16.2$ \\
Current alcohol drinker & $0.0 \%$ \\
Former alcohol drinker & $7.0 \%$ \\
Current smoker & $2.2 \%$ \\
Former smoker & $37.5 \%$ \\
Systolic blood pressure (mm Hg) & $135.6 \pm 17.1$ \\
Diastolic blood pressure (mm Hg) & $85.8 \pm 11.8$ \\
Diabetes mellitus & $34.4 \%$ \\
Hypertension & $71.4 \%$ \\
Dyslipidemia & $41.1 \%$ \\
Cardiovascular diseases & $7.6 \%$ \\
Obstructive sleep apnea & $38.4 \%$ \\
Fasting plasma glucose (mg/dL) & $111.2 \pm 35.9$ \\
Glycated hemoglobin (\%) & $6.3 \pm 1.2$ \\
Total cholesterol (mg/dL) & $186.3 \pm 36.1$ \\
HDL-cholesterol (mg/dL) & $45.3 \pm 12.2$ \\
Triglycerides (mg/dL) & $161.0 \pm 81.0$ \\
LDL-cholesterol (mg/dL) & $109.2 \pm 32.6$ \\
AST (U/L) & $20(17-25)$ \\
ALT (U/L) & $23(17-32)$ \\
\hline
\end{tabular}

Data are presented as mean \pm standard deviation, median (interquartile range), or proportion $\mathrm{n}(\%) . A L T$ : alanine transaminase; AST: aspartate transaminase; $B M I$ : body mass index. HDL: high-density lipoprotein; $L D L$ : low-density lipoprotein. Excess weight was calculated with a BMI of $25 \mathrm{~kg} / \mathrm{m}^{2}$ as a reference for ideal body weight.
Table 2

Liver biopsy data analysis $(\mathrm{N}=227)$.

\begin{tabular}{ll}
\hline Liver histology & $\mathrm{N}(\%)$ \\
\hline Normal & $74(32.6 \%)$ \\
NAFLD & $153(67.4 \%)$ \\
Steatosis & $41(18 \%)$ \\
Mild & $29(12.8 \%)$ \\
Moderate & $10(4.4 \%)$ \\
Severe & $2(0.9 \%)$ \\
Steatohepatitis & a \\
Mild & $112(49.3 \%)$ \\
Moderate & $103(44.9 \%)$ \\
Severe & $8(3.5 \%)$ \\
Fibrosis & $2(0.9 \%)$ \\
Periportal & $55(24.2 \%)$ \\
Perisinusoidal & $19(8.4 \%)$ \\
Periportal and perisinusoidal & $25(11 \%)$ \\
Bridging fibrosis & $6(2.6 \%)$ \\
Portal to portal & $2(0.9 \%)$ \\
Portal to center & $0(0.0 \%)$ \\
Cirrhosis & $2(0.9 \%)$ \\
\hline
\end{tabular}

${ }^{a}$ Steatohepatitis classified according to the percentage of fat infiltration of liver parenchyma. NAFLD: nonalcoholic fatty liver disease.

Table 3

Ultrasound performance in diagnosing nonalcoholic fatty liver disease compared with liver histology.

\begin{tabular}{|c|c|c|c|}
\hline \multirow[t]{4}{*}{ Ultrasound } & \multirow{4}{*}{$\begin{array}{l}\text { Positive } \\
\text { Negative }\end{array}$} & \multicolumn{2}{|c|}{ Liver biopsy } \\
\hline & & Positive & Negative \\
\hline & & 136 & 41 \\
\hline & & 17 & 33 \\
\hline \multicolumn{2}{|c|}{ NAFLD prevalence } & & $67.4 \%$ \\
\hline \multicolumn{2}{|c|}{ Sensitivity } & & $88.9 \%$ \\
\hline \multicolumn{2}{|c|}{ Specificity } & & $44.6 \%$ \\
\hline \multicolumn{2}{|c|}{ Positive predictive value } & & $76.8 \%$ \\
\hline \multicolumn{2}{|c|}{ Negative predictive value } & & $66.0 \%$ \\
\hline \multicolumn{2}{|c|}{ Positive likelihood ratio ${ }^{\mathrm{a}}$} & & $1.6(1.30-1.98)$ \\
\hline \multicolumn{2}{|c|}{ Negative likelihood ratio ${ }^{a}$} & & $0.25(0.15-0.42)$ \\
\hline \multicolumn{2}{|c|}{ Accuracy $^{\text {a }}$} & & $74.5 \%(68.3 \%-80 \%)$ \\
\hline
\end{tabular}

${ }^{a}$ Data are presented as mean and 95\% confidence interval. NAFLD: nonalcoholic fatty liver disease.

regarding the diagnostic approach to NAFLD [17]. In different clinical settings, evaluating the diagnostic properties of many tests compared with liver histology in a sufficiently large sample is challenging [18].

Despite the high prevalence of NAFLD diagnosed by liver biopsy among patients included in this study (67.4\%), this finding was comparable to those of several reports in an apparently similar population $[19,20]$. Higher prevalences than those observed in this study were also reported [21,22]. Variability in patient characteristics, health care systems, measurement methods, and determinants of steatosis, especially alcohol and viruses, could be responsible for these differences [20]. However, these numbers are definitively higher than those observed in the general population [23].

Despite the high prevalence of NASH in liver biopsies, most patients had liver enzymes within normal limits, which may seem unusual. In fact, prevalence studies based on elevated liver enzymes systematically underestimate the true prevalence of NASH compared with studies based on imaging tests [2]. Moreover, the degree of aminotransferase elevation does not correlate with the diagnosis of NASH, severity of fibrosis, or severity of inflammation [24]. Even patients with severe NASH may have normal liver enzymes [25].

A previous study in a similar setting reported a lower sensitivity and a much higher specificity than those reported herein [26]. Possibly, the number of patients with a negative biopsy result included in that study (11 and 16, respectively) was very low to estimate ultrasound specificity. Moreover, overall agreement between the two tests is influenced by the prevalence of disease, which is undoubtedly different between 


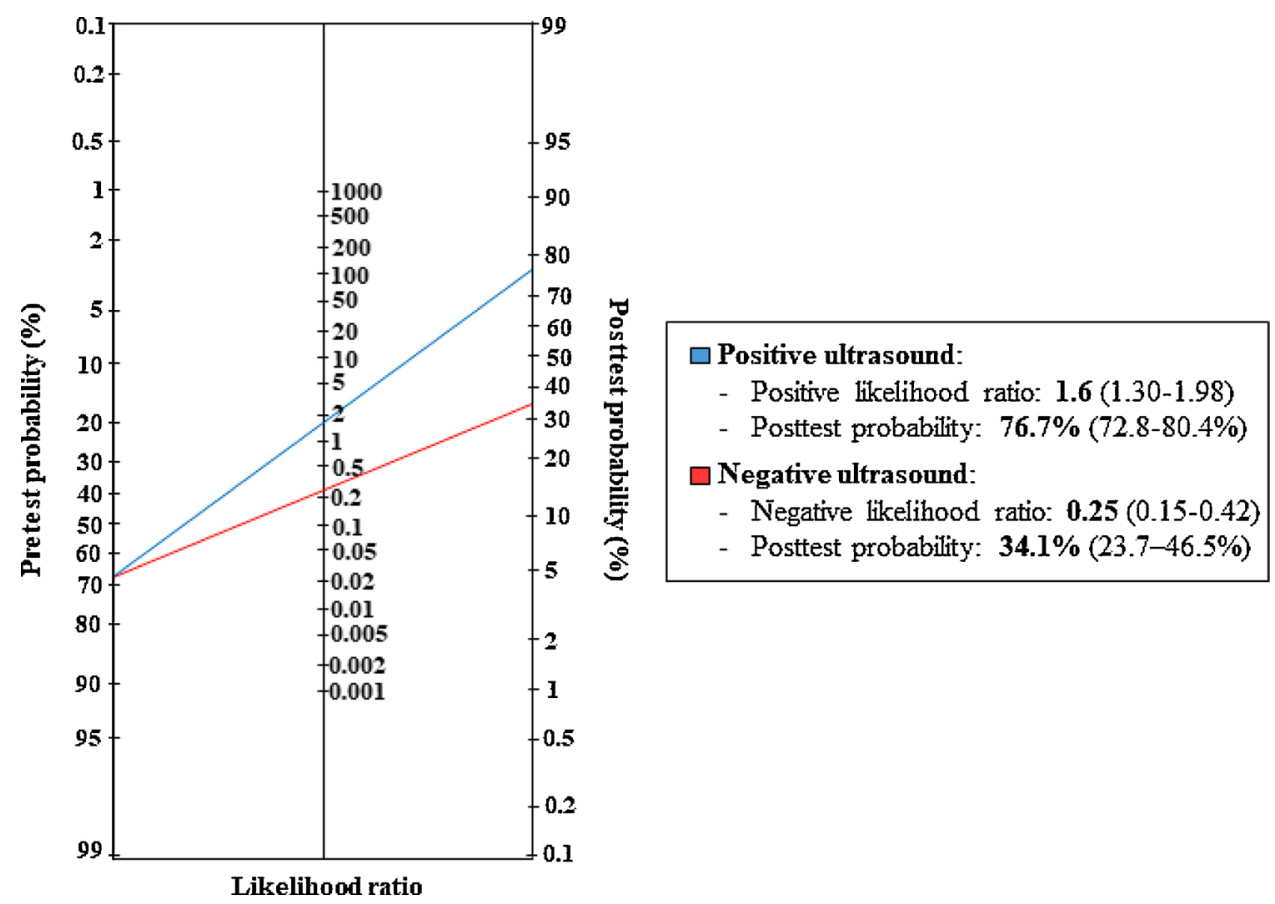

Fig. 2. Fagan nomograph with pretest and posttest probabilities of nonalcoholic fatty liver disease within a $95 \%$ confidence interval.

studies, as stated above [27,28]. Therefore, as our findings are derived from a much larger sample, it is possible that ultrasound specificity is not that high.

A large systematic review with meta-analysis [10] summarized a similar sensitivity (84.8\%) but a much higher specificity (93.6\%) for ultrasound in diagnosing liver steatosis. Nevertheless, most participants of the studies included in that meta-analysis were patients with liver disease or suspected liver disease, thereby differing from the patients of our study. Also, data on patients' BMI were not described. Moreover, our sample consisted mostly of patients with mild steatosis. Histological criteria for defining the severity of fat deposition were also different: in the meta-analysis, mild fat infiltration (up to $20 \%$ or $30 \%$ of liver parenchyma) was considered absence of steatosis.

In the evaluation of diagnostic accuracy of a medical test, participants are expected to undergo both the index and reference tests within a short period. This would avoid biases caused by changes in their true disease status, which can also affect the diagnostic accuracy of the index test [29]. This information was not available for all patients in our database and was not provided by other studies [22,23]. An ultrasound is usually ordered at the time patients start initial examinations to be considered eligible for bariatric surgery. Although bariatric surgery is an effective treatment for class II and III obesity and is associated with long-term improvement in obesity-related diseases [30], the demand is greater than the capacity in several countries [31]. A recent study conducted by our group showed that the median waiting time for surgery was 30 (21-41) months [32]. Considering the preoperative management of participants, the long interval between tests could have led to an increase in false-positive rate. Despite this potential conservative bias, the index test showed a good sensitivity.

Other than methodological differences, the low specificity observed in this study could also be explained by the qualitative nature of ultrasound [14]. Considering the widespread knowledge that NAFLD has an extremely high prevalence among patients with severe obesity, the appraisal of an operator-dependent test (such as ultrasound) may entail a high risk of bias. Hence, our hypothesis is that ultrasound operators tend to overestimate ultrasonographic findings in morbidly obese individuals, which increases the false-positive rate. Moreover, there is a wide variation in ultrasound parameters for assessing fatty liver, and they were not described in detail in the studies.

The present study has some limitations. The uneven distribution of NASH lesions throughout the liver parenchyma can lead to sampling error, misdiagnosis, and staging inaccuracies [33]. The qualitative nature of ultrasound assessment leads to limited intra- and interobserver variability [34]. Other ultrasound techniques (e.g., Doppler and histogram) that would have allowed a more objective quantification of fat infiltration in the liver parenchyma were not included. Also, the estimated accuracy may have been lowered by the long time between ultrasound and intraoperative biopsy. The strengths of this study include the assessment of a large number of liver biopsies as the gold standard, the evaluation of liver biopsies performed by only one expert, the ultrasound operator's unawareness of biopsy results, and the representative sample of patients with normal liver histology.

This study highlights some concerns about the diagnostic performance of ultrasound in patients with class II and III obesity. The estimated sensitivity of $88.9 \%$ supports the widespread recommendation of ultrasound as the first-line test for NAFLD screening in these patients. However, unlike previous studies, our estimated $44.6 \%$ specificity points to an important impact of severe obesity in ultrasound qualitative assessment of the liver parenchyma. In summary, once ultrasound alone is not enough to state a NAFLD diagnosis in patients with class II and III obesity, further steps following ultrasound must be included in NAFLD investigation.

\section{Funding information}

This study was supported by Conselho Nacional de Desenvolvimento Científico e Tecnológico (CNPq), by Coordenação de Aperfeiçoamento de Pessoal de Nível Superior - Brasil (CAPES) - Finance Code 001, and by Fundo de Incentivo à Pesquisa e Eventos (FIPE) of Hospital de Clínicas de Porto Alegre.

\section{Conflicts of interest}

The authors declare that they have no conflicts of interest to disclose. 


\section{Ethical approval statement}

All procedures performed in studies involving human participants were in accordance with the ethical standards of the institutional and/or national research committee and with the 1964 Helsinki Declaration and its later amendments or comparable ethical standards.

\section{Informed consent statement}

For retrospective studies, formal consent is not required.

\section{CRediT authorship contribution statement}

Gabriel Leivas: Conceptualization, Methodology, Investigation, Writing - original draft. Clara K. Maraschin: Conceptualization, Investigation, Writing - original draft, Visualization. Carina A. Blume: Methodology, Validation, Formal analysis, Investigation, Writing original draft, Writing - review \& editing. Gabriela H. Telo: Validation, Supervision, Writing - review \& editing. Manoel R.M. Trindade: Investigation, Resources, Supervision. Eduardo N. Trindade: Validation, Investigation, Resources, Supervision. Vinicius V. Diemen: Methodology, Validation, Investigation, Resources, Writing - original draft. Carlos Thadeu S. Cerski: Methodology, Validation, Investigation, Resources, Writing - original draft. Beatriz D. Schaan: Conceptualization, Methodology, Validation, Resources, Writing - review \& editing, Project administration, Funding acquisition.

\section{References}

[1] Younossi ZM, Blissett D, Blissett R, Henry L, Stepanova M, Younossi Y, et al. The economic and clinical burden of nonalcoholic fatty liver disease in the United States and Europe. Hepatology 2016;64(5):1577-86. https://doi.org/10.1002/ hep. 28785.

[2] Younossi ZM, Koenig AB, Abdelatif D, Fazel Y, Henry L, Wymer M. Global epidemiology of nonalcoholic fatty liver disease-meta-analytic assessment of prevalence, incidence, and outcomes. Hepatology 2016;64(1):73-84. https://doi. org/10.1002/hep.28431.

[3] Dai W, Ye L, Liu A, Wen SW, Deng J, Wu X, et al. Prevalence of nonalcoholic fatty liver disease in patients with type 2 diabetes mellitus: a meta-analysis. Medicine (Baltimore) 2017;96(39):e8179. https://doi.org/10.1097/ MD.0000000000008179.

[4] Machado M, Marques-Vidal P, Cortez-Pinto H. Hepatic histology in obese patients undergoing bariatric surgery. J Hepatol 2006;45(4):600-6. https://doi.org/ 10.1016/j.jhep.2006.06.013.

[5] Starley BQ, Calcagno CJ, Harrison SA. Nonalcoholic fatty liver disease and hepatocellular carcinoma: a weighty connection. Hepatology 2010;51(5):1820-32. https://doi.org/10.1002/hep.23594.

[6] Estes C, Razavi H, Loomba R, Younossi Z, Sanyal AJ. Modeling the epidemic of nonalcoholic fatty liver disease demonstrates an exponential increase in burden of disease. Hepatology 2018;67(1):123-33. https://doi.org/10.1002/hep.29466.

[7] Yoneda M, Imajo K, Takahashi H, Ogawa Y, Eguchi Y, Sumida Y, et al. Clinical strategy of diagnosing and following patients with nonalcoholic fatty liver disease based on invasive and noninvasive methods. J Gastroenterol 2018;53(2):181-96. https://doi.org/10.1007/s00535-017-1414-2.

[8] Araújo AR, Rosso N, Bedogni G, Tiribelli C, Bellentani S. Global epidemiology of non-alcoholic fatty liver disease/non-alcoholic steatohepatitis: what we need in the future. Liver Int 2018;38 Suppl 1:47-51. https://doi.org/10.1111/liv.13643.

[9] European Association for the Study of the Liver (EASL), European Association for the Study of Diabetes (EASD), European Association for the Study of Obesity (EASO). EASL-EASD-EASO clinical practice guidelines for the management of nonalcoholic fatty liver disease. J Hepatol 2016;64(6):1388-402. https://doi.org/ 10.1016/j.jhep.2015.11.004.

[10] Hernaez R, Lazo M, Bonekamp S, Kamel I, Brancati FL, Guallar E, et al. Diagnostic accuracy and reliability of ultrasonography for the detection of fatty liver: a metaanalysis. Hepatology 2011;54(3):1082-90. https://doi.org/10.1002/hep.24452.

[11] Shalhub S, Parsee A, Gallagher SF, Haines KL, Willkomm C, Brantley SG, et al. The importance of routine liver biopsy in diagnosing non-alcoholic steatohepatitis in bariatric patients. Obes Surg 2004;14:54-9. https://doi.org/10.1381/ 096089204772787293.

[12] Physical status: the use and interpretation of anthropometry. Report of a WHO Expert Committee. World Health Organ Tech Rep Ser 1995;854:1-452.
[13] Bossuyt PM, Reitsma JB, Bruns DE, Gatsonis CA, Glasziou PP, Irwig L, et al. STARD 2015: an updated list of essential items for reporting diagnostic accuracy studies. BMJ 2015;351:h5527. https://doi.org/10.1136/bmj.h5527.

[14] Castera L, Friedrich-Rust M, Loomba R. Noninvasive assessment of liver disease in patients with nonalcoholic fatty liver disease. Gastroenterology 2019;156(5). https://doi.org/10.1053/j.gastro.2018.12.036. 1264-1281.e4.

[15] Kleiner DE, Brunt EM, Van Natta M, Behling C, Contos MJ, Cummings OW, et al. Design and validation of a histological scoring system for nonalcoholic fatty liver disease. Hepatology 2005;41(6):1313-21. https://doi.org/10.1002/hep.20701.

[16] Chalasani N, Younossi Z, Lavine JE, Charlton M, Cusi K, Rinella M, et al. The diagnosis and management of nonalcoholic fatty liver disease: practice guidance from the American Association for the Study of Liver Diseases. Hepatology 2018;67 (1):328-57. https://doi.org/10.1002/hep.29367.

[17] Lee SS, Park SH. Radiologic evaluation of nonalcoholic fatty liver disease. World J Gastroenterol 2014;20(23):7392-402. https://doi.org/10.3748/wjg.v20.i23.7392.

[18] Papatheodoridi M, Cholongitas E. Diagnosis of non-alcoholic fatty liver disease (NAFLD): current concepts. Curr Pharm Des 2018;24(38):4574-86. https://doi. org/10.2174/1381612825666190117102111.

[19] Ooi GJ, Burton PR, Bayliss J, Raajendiran A, Earnest A, Laurie C, et al. Effect of body mass index, metabolic health and adipose tissue inflammation on the severity of non-alcoholic fatty liver disease in bariatric surgical patients: a prospective study. Obes Surg 2019;29(1):99-108. https://doi.org/10.1007/s11695-018-34792.

[20] Schwenger KJP, Fischer SE, Jackson TD, Okrainec A, Allard JP. Non-alcoholic fatty liver disease in morbidly obese individuals undergoing bariatric surgery: prevalence and effect of the pre-bariatric very low calorie diet. Obes Surg 2018;28 (4):1109-16. https://doi.org/10.1007/s11695-017-2980-3.

[21] Souto KP, Meinhardt NG, Ramos MJ, Ulbrich-Kulkzynski JM, Stein AT, Damin DC. Nonalcoholic fatty liver disease in patients with different baseline glucose status undergoing bariatric surgery: analysis of intraoperative liver biopsies and literature review. Surg Obes Relat Dis 2018;14(1):66-73. https://doi.org/10.1016/j. soard.2017.09.527.

[22] Morita S, Neto Dde S, Morita FH, Morita NK, Lobo SM. Prevalence of non-alcoholic fatty liver disease and steatohepatitis risk factors in patients undergoing bariatric surgery. Obes Surg 2015;25(12):2335-43. https://doi.org/10.1007/s11695-0151696-5.

[23] Shouhed D, Steggerda J, Burch M, Noureddin M. The role of bariatric surgery in nonalcoholic fatty liver disease and nonalcoholic steatohepatitis. Expert Rev Gastroenterol Hepatol 2017;11(9):797-811. https://doi.org/10.1080/ 17474124.2017 .1355731$.

[24] Verma S, Jensen D, Hart J, Mohanty SR. Predictive value of ALT levels for nonalcoholic steatohepatitis (NASH) and advanced fibrosis in non-alcoholic fatty liver disease (NAFLD). Liver Int 2013;33(9):1398-405. https://doi.org/10.1111/ liv.12226.

[25] Noureddin M, Vipani A, Bresee C, Todo T, Kim IK, Alkhouri N, et al. NASH leading cause of liver transplant in women: updated analysis of indications for liver transplant and ethnic and gender variances. Am J Gastroenterol 2018;113(11): 1649-59. https://doi.org/10.1038/s41395-018-0088-6.

[26] Mottin CC, Moretto M, Padoin AV, Swarowsky AM, Toneto MG, Glock L, et al. The role of ultrasound in the diagnosis of hepatic steatosis in morbidly obese patients. Obes Surg 2004;14(5):635-7. https://doi.org/10.1381/096089204323093408.

[27] Gart JJ, Buck AA. Comparison of a screening test and a reference test in epidemiologic studies II. A probabilistic model for the comparison of diagnostic tests. Am J Epidemiol 1966;83(3):593-602. https://doi.org/10.1093/ oxfordjournals.aje.a120610.

[28] Holterman AX, Guzman G, Fantuzzi G, Wang H, Aigner K, Browne A, et al. Nonalcoholic fatty liver disease in severely obese adolescent and adult patients. Obesity (Silver Spring) 2013;21(March (3)):591-7. https://doi.org/10.1002/ oby. 20174

[29] Umemneku Chikere CM, Wilson K, Graziadio S, Vale L, Allen AJ. Diagnostic tes evaluation methodology: a systematic review of methods employed to evaluate diagnostic tests in the absence of gold standard — an update. PLoS One 2019;14 (10). https://doi.org/10.1371/journal.pone.0223832. e0223832.

[30] Jakobsen GS, Småstuen MC, Sandbu R, Nordstrand N, Hofsø D, Lindberg M, et al. Association of bariatric surgery vs medical obesity treatment with long-term medical complications and obesity-related comorbidities. JAMA 2018;319(3): 291-301. https://doi.org/10.1001/jama.2017.21055.

[31] Alvarez R, Bonham AJ, Buda CM, Carlin AM, Ghaferi AA, Varban OA. Factors associated with long wait times for bariatric surgery. Ann Surg 2019;270(6): 1103-9. https://doi.org/10.1097/SLA.0000000000002826.

[32] Blume CA, Brust-Renck PG, Rocha MK, Leivas G, Neyeloff JL, Anzanello MJ, et al. Development and validation of a predictive model of success in bariatric surgery. Obes Surg 2021;31(3):1030-7. https://doi.org/10.1007/s11695-020-05103-0.

[33] Ratziu V, Charlotte F, Heurtier A, Gombert S, Giral P, Bruckert E, et al. Sampling variability of liver biopsy in nonalcoholic fatty liver disease. Gastroenterology 2005;128(7):1898-906. https://doi.org/10.1053/j.gastro.2005.03.084.

[34] Strauss S, Gavish E, Gottlieb P, Katsnelson L. Interobserver and intraobserver variability in the sonographic assessment of fatty liver. AJR Am J Roentgenol 2007; 189(6):W320-3. https://doi.org/10.2214/AJR.07.2123. 